\title{
A pulsed field gradient spin echo NMR spectrometer for diffusion coefficient measurements
}

\author{
BHASKAR COLLUROY ${ }^{\dagger}$, G MANGAMMA and S V BHAT \\ Department of Physics, Indian Institute of Science, Bangalore 560012, India \\ ${ }^{\dagger}$ Present address: B M S College of Engineering, Bangalore 560019, India
}

MS received 25 February 1988; revised 22 April 1988

\begin{abstract}
A pulsed field gradient spin echo NMR spectrometer has been assembled by interfacing a programmable pulse generator and a data acquisition system designed and fabricated in our laboratory with other imported units. Calibration results of the magnetic field gradients are presented.
\end{abstract}

Keywords. NMR spin echoes; pulsed field gradients; diffusion coefficients; fast ionic conductors.

PACS No. $76 \cdot 60$

\section{Introduction}

An accurate knowledge of diffusion coefficients is essential in diverse fields of science like physics, chemistry and biology. Currently, the measurement of diffusion coefficient is of interest in the study of fast ionic conductors, hydrogen in metal systems, liquid crystals, micro-emulsion systems and transport across membranes. Of the different methods available for the purpose, like the radio-tracer method, measurement of conductivity and the like, NMR methods have been found to be very useful. Study of the linewidth variation with temperature in the region of motional narrowing and the study of the temperature dependence of the relaxation times have been the most frequently used NMR procedures until recently. However, to extract the diffusion coefficients from these experiments one needs to rely upon some particular model for diffusion and many a time it is found that one obtains inconsistent values for the potential barrier and the pre-exponential factors, while trying to fit the temperature dependence to an Arrhenius type relation (Boyce and Huberman 1979).

A better way to determine the diffusion coefficient $D$ is by the study of the spin echoes under magnetic field gradients, especially with the ingenious technique of pulsed field gradients, first devised by Stejskal and Tanner (1975). This technique, to be described briefly in the following sections, makes use of the phase of the Larmor precession of the nuclear spins in a magnetic field as a probe, conceivably the most non-perturbing probe one can use to monitor the transport of atoms or ions through the sample.

We have recently assembled a pulsed field gradient NMR spin echo spectrometer in our laboratory, mainly to study diffusion processes in fast ionic conductors. However, the spectrometer is easily adaptable for the measurement of $D$ in other systems 
mentioned earlier. While putting together this spectrometer, our objectives have been two-fold; first, to develop and make use of indigenous knowhow wherever possible and second, to import state-of-the-art units whose construction is not possible because of the non-availability of either the components or the infrastructure. While interfacing the imported units to the indigenously built ones, valuable training of manpower was expected to be obtained. A preliminary report of this work was presented in a symposium (Bhaskar et al 1986).

In what follows, $\S 2$ describes the basic principles of measurement of diffusion coefficient using the pulsed field gradient (PFG) spin echo technique. A general description of the spectrometer presented in $\$ \S 3,4$ and 5 gives details of the pulse programmer and the data acquisition system respectively. In $\S 6$ we discuss the calibration of the gradients and the conclusions in $\$ 7$.

\section{The PFG spin echo technique}

It has been known since the early days of NMR that one can measure the self-diffusion coefficients very accurately using the NMR spin-echo technique (Hahn 1950). The spin echo amplitude in the presence of a field gradient $g_{0}$ in a given direction is given by

$$
M_{g}=M(\infty) \exp \left(-2 \tau / T_{2}-2 / 3 \gamma^{2} g_{0}^{2} D \tau^{3}\right)
$$

where $M(\infty)$ is the amplitude of the free induction decay (FID), $M_{g}$ is the spin echo amplitude after a delay of $2 \tau$ after the $180^{\circ}$ pulse in the $90^{\circ}-\tau-180^{\circ}$ spin-echo sequence, $T_{2}$ is the spin-spin relaxation time and $\gamma$ is the gyromagnetic ratio of the nucleus. The echo amplitude in the absence of the gradient is

$$
M_{0}=M(\infty) \exp \left(-2 \tau / T_{2}\right)
$$

The diffusion coefficient $D$ can therefore be determined by the ratio

$$
\ln \left(M_{g} / M_{0}\right)=-2 / 3 \gamma^{2} g_{0}^{2} D \tau^{3}
$$

In this method, acquisition of the echo is carried out in the presence of the gradient, which broadens the echo. The problems of the occurrence of this artificial broadening as well as those of the requirements of high transmitter power and broad band receivers

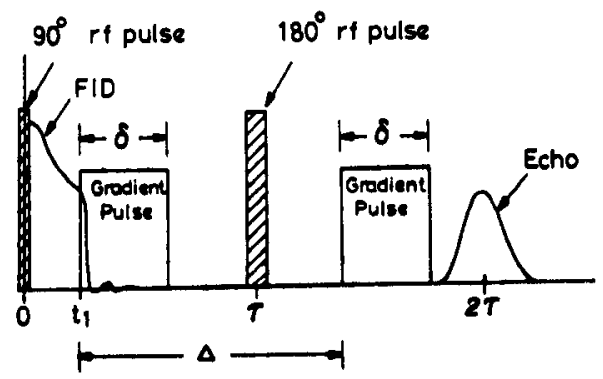

Figure 1. The pulsed field gradient spin echu method: a pair of gradient pulses each of width $\delta$, strength $g$ and separated from each other by a time $\Delta$ are placed between the $\pi / 2$ and the $\pi$ r.f. pulses and the $\pi$ r.f. pulse and the echo. 
can be eliminated by the method of pulsed gradients. Figure 1 illustrates the principle of this method. Instead of the steady gradient, two gradient pulses of strength $g$, duration $\delta$ and separation $\Delta$ are applied after the $90^{\circ}$ and the $180^{\circ}$ r.f. pulses. It can be shown (Stejskal and Tanner 1975) that for this experiment the ratio of the echo amplitudes in the presence and absence of the gradient pulses respectively is given by

$$
\ln \left(M_{g} / M_{0}\right)=-\gamma^{2} D g^{2} \delta^{2}(\Delta-1 / 3 \delta) .
$$

Since there is no gradient present while the echo is observed, there is no broadening of the echo and therefore higher magnitudes of the gradient can be used enabling the measurement of much smaller diffusion coefficients.

\section{General description of the spectrometer}

Figure 2 presents the block diagram of the NMR spectrometer. The magnet is a Bruker BMN 20 electromagnet with adjustable gap and its homogeneity improved (to $\sim 3$ parts in $10^{6}$ ) by using special ring-shim polecaps. The BMN $155 / 45$ power supply to the magnet can deliver a maximum power of $7 \mathrm{~kW}$ corresponding to a field of $18 \mathrm{k}$ Gauss for a gap of $5 \mathrm{~cm}$. The current is regulated to 1 part in $10^{6}$. The r.f. source is a Marconi Instruments signal generator with an output level of $1 \mathrm{~V}$ peak-to-peak over a range of $10 \mathrm{kHz}$ to $89 \mathrm{MHz}$. The r.f. is mixed with the gating pulses generated in pulse programmer (to be described below) in a double balanced mixer (Anzac MD 143). The pulsed r.f. is first amplified to $\sim 20 \mathrm{~W}$ in a broad band driver amplifier before being amplified further in a tuned power amplifier which can deliver power levels upto $1.5 \mathrm{~kW}$ into a $50 \Omega$ load. The output of the power amplifier is fed to the probe head incorporating the tank coil via a tunable probearm. The NMR signal is amplified in the preamplifier before being detected in the receiver which can operate either in the diode detection mode or the phase sensitive detection mode. The signal from the receiver can be displayed on the oscilloscope and/or digitized/signal-averaged in the data acquisition system. The main units designed and fabricated in our laboratory are the programmable pulse generator (PPG) and the data acquisition system (DAS) apart from the low voltage regulated power supplies and the interfacing units. The PPG and the DAS are described below.

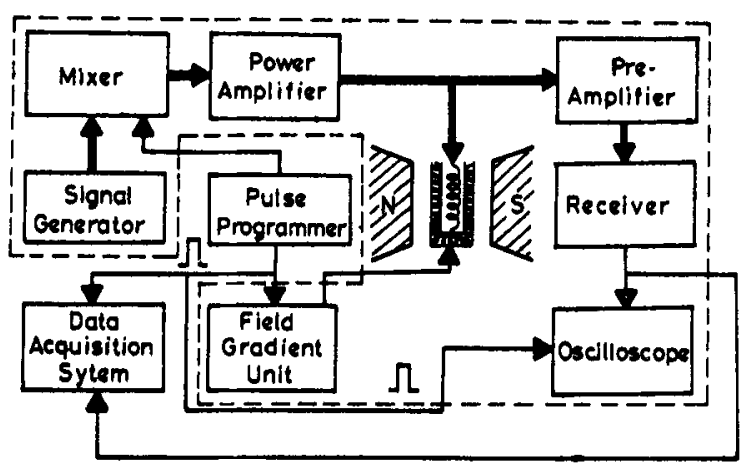

Figure 2. Block diagram of the PFG spin echo NMR spectrometer. The imported units are shown enclosed in dashed lines. 


\section{Programmable pulse generator}

We have designed and built a simple and inexpensive pulse programmer based on a locally available 8085 microprocessor kit making use of programmable 8253 counters. With this pulse programmer most of the popular pulse schemes in NMR like $n 90^{\circ}-\tau$ $90^{\circ}, 180^{\circ}-\tau-90^{\circ}$ and $90^{\circ}-\tau-180^{\circ}$ sequences can be generated through software control as can also the trigger pulses for the oscilloscope and the data acquisition system, without requiring any hardware changes. The pulse programmer has the following essential specifications: pulse width 1-50 $\mathrm{S}$; delay between the 1 st and the 2nd pulse sequences; $5 \mu \mathrm{S}-5 \mathrm{~S}$ and the rate of repetition of the pulse cycle; $500 \mu \mathrm{S}$ to a few minutes. The details of the pulse programmer have already been published (Mangamma et al 1987).

\section{Data acquisition system}

The data acquisition system (DAS) comprises of a sample and hold $(\mathrm{S} / \mathrm{H})$ circuit and an analog-to-digital converter. The $\mathrm{S} / \mathrm{H}$ circuit is constructed using the analog switch $\mathrm{CD}$ 4066. The analog-to-digital (A/D) converter used is a National Semiconductor 8-bit ADC 0820 with a conversion time $2.5 \mu \mathrm{S}$ in the read-mode and the maximum input voltage handling capacity of 5 volts. The DAS is triggered by either the $90^{\circ}$ or the $180^{\circ}$ pulse from the pulse generator. The programmable features of the DAS are (a) the delay between the trigger and the sampling window, (b) the width of the sampling window and (c) the number of repetitive scans for signal averaging.

The software employed is of a descriminatory type with the capability to distinguish between spurious and genuine data. Before acquiring the final signal-averaged data for processing, the unit samples a certain number (which can be specified for each run; typically $\sim 20$ ) of trial signals and finds their average. Then each of the data point is compared with this average value and is retained only if it falls within a certain limit (which can be specified and is typically $\pm 5 \%$ ) of the average value. This way, spurious data points are rejected.

Figure 3 presents the schematic diagram of the DAS.

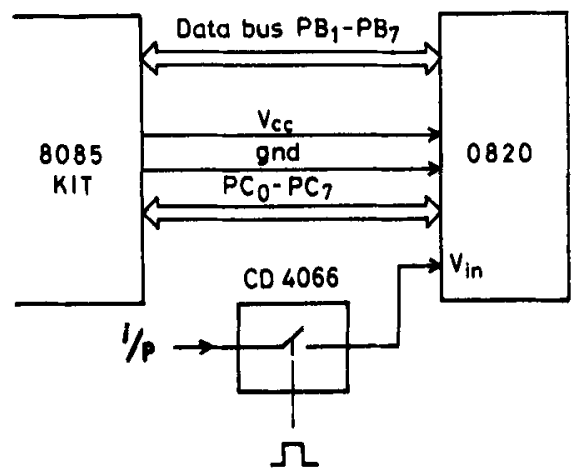

Figure 3. Schematic diagram of the data acquisition system. 


\section{Field gradient unit and calibration of field gradients}

The field gradients are generated through a pair off anti-Helmholtz coils mounted on the two sides of the NMR probe head. The Bruker B-Z18B field gradient unit which we use for this purpose has provisions for generating both steady and pulsed field gradients with adjustable strengths of the gradients. The width of the pulsed gradients and the separation between them can also be varied.

As can be seen from equations (3) and (4), it is necessary to determine the magnitudes of the field gradients (steady or pulsed) accurately for precise evaluation of the diffusion coefficients. Many methods are available (Murday 1973) for this purpose, namely: (i) actual measurement of the magnetic field at different points using a field probe, (ii) experiment on a sample with a known diffusion coefficient, (iii) analysis of the shape of the free induction decay, (iv) calculation of the field gradient using the Biot-Savarat law and (v) the use of a split pair sample holder and the study of the shift in the echo position in the absence of one of the gradient pulses (Bhat 1984). We have calibrated the steady gradient by studying the shape of the FID and obtain a maximum gradient of 20 Gauss $/ \mathrm{cm}$. The pulsed gradients have been calibrated by utilizing the known diffusion coefficients at room temperature of $n$-decanol $\left(D=8.3 \times 10^{-7} \mathrm{~cm}^{2} / \mathrm{s}\right)$. Figure 4 shows the dependence of the echo intensity on the duration $\delta$, and separation $\Delta$ of the gradient pulses for a dial setting of 500 of the pulsed gradient unit. Utilizing the known D of Ndecanol and $\gamma$ for protons, the gradient value for this dial setting is calculated from equation (4).

This calibration is checked by determining the diffusion coefficient of distilled water. For the same dial setting figure 5 shows the dependence of echo attenuation of $\delta$ and $\Delta$. Making use of the slope of the graph, $D$ of distilled water is calculated to be $2 \cdot 1 \times 10^{-5} \mathrm{~cm}^{2} / \mathrm{s}$ which compares well with the reported value (Tsai et al 1983), $2 \cdot 3 \times 10^{-5} \mathrm{~cm}^{2} / \mathrm{s}$.

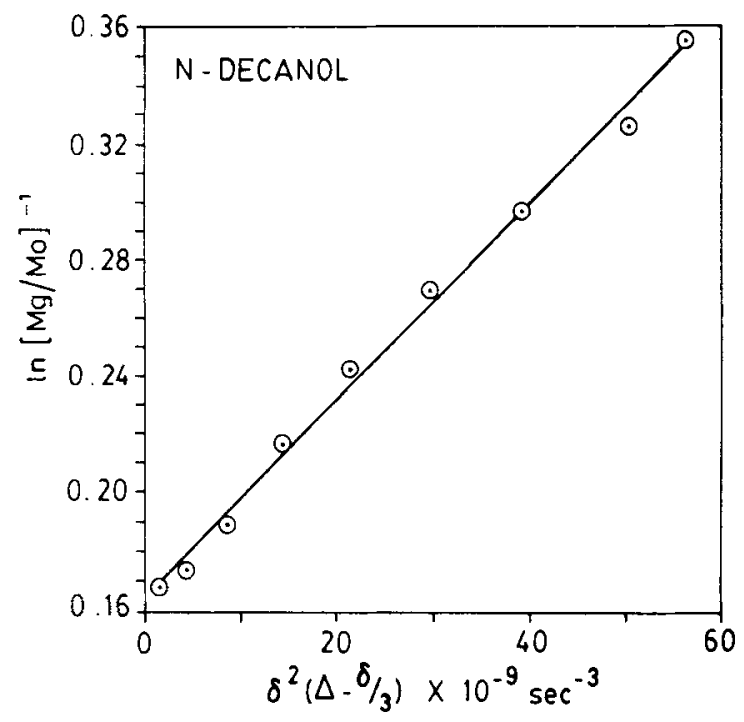

Figure 4. Dependence of echo intensity on the gradient separation $\Delta$ and width $\delta$ for $N$ decanol, for a dial setting of 500 . 


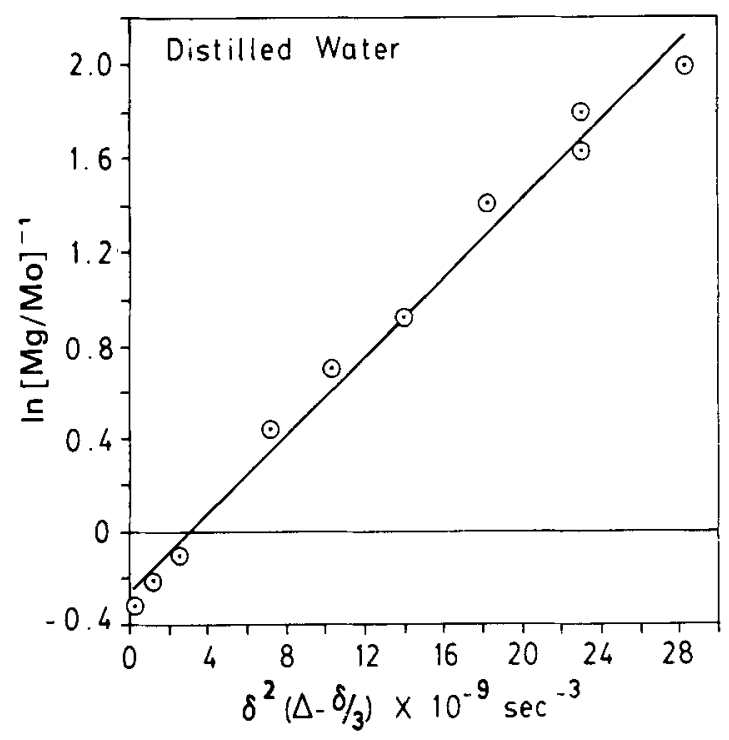

Figure 5. Dependence of echo intensity on the gradient separation $\Delta$ and width $\delta$ for distilled water for a dial setting of 500 .

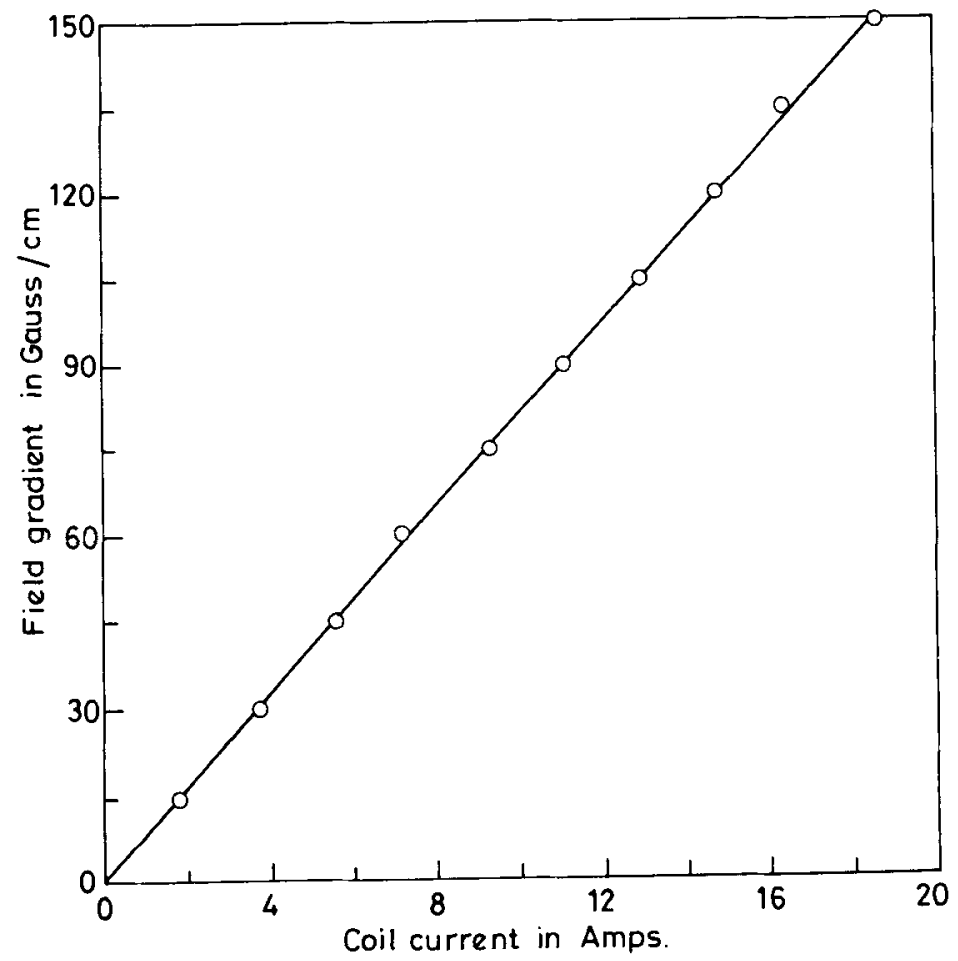

Figure 6. Plot of the magnitude of the pulsed field gradient against the coil current. 
Such experiments have been carried out for various dial settings corresponding to different currents through the gradient coils. Figure 6 shows the gradient magnitudes thus determined, corresponding to different currents through the gradient coils.

\section{Conclusions}

In conclusion, we have assembled a pulsed field gradient spin echo NMR spectrometer by interfacing home-built microprocessor-based pulse programmer and data acquisition system to imported r.f. units and an electromagnet. We have so far utilized the spectrometer for studies on the temperature and frequency dependences of relaxation times in certain protonic conductors (Mangamma and Bhat 1986). The measurements of the diffusion coefficients of these substances are now in progress.

\section{Acknowledgements}

Financial assistance from the Department of Science and Technology through a project grant to one of us (SVB) is gratefully acknowledged. The authors would also like to thank Mr Gajanan, Ms Kalyani Murthy and Ms Anuradha Kadba for their assistance at various stages of this project.

\section{References}

Bhat S V 1984 Curr. Sci. 53418

Bhaskar C, Mangamma G and Bhat S V 1986 National symposium on instrumentation NSI-11, Hyderabad, October 1986

Boyce J B and Huberman B A 1979 Phys. Rep. 51189

Hahn E L 1950 Phys. Rev. 80580

Mangamma G, Balakrishnan N and Bhat S V 1987 J. Phys. E20 100

Mangamma G and Bhat S V 1986 Proc. Solid State Phys. Symp. C29 207

Murday J S 1973 J. Magn. Res. 1011

Stejskal E D and Tanner J E 1975 J. Chem. Phys. 42288

Tsai Y T, Halperin W P and Whitmore D H 1983 J. Solid State Chem. 50263 